\title{
Clinical analysis on nosocomial pulmonary infection in patients with indwelling tracheal tube
}

\author{
Jin Guo, Shuna Shen*, Chunyan Qu, Cuiping Yan \\ Comprehensive Surgery Department, The Third Affiliated Hospital of Inner Mongolia Medical University, China
}

Received: August 30, 2015

DOI: $10.14725 /$ dcc.v2n4p22
Accepted: November 12, $2015 \quad$ Online Published: December 10, 2015

URL: http://dx.doi.org/10.14725/dcc.v2n4p22

\begin{abstract}
Tracheal intubation by tracheotomy or by placing a tracheal tube through larynx into trachea, is not only one of important measures which are taken to rescue critically ill patients with respiratory failure etc., but a commonly used method to prevent patients from airway obstruction in the postoperative period and facilitate mechanical ventilation. Meanwhile, the incidence rate of pulmonary infection, a complication caused by indwelling tracheal tubes, especially the incidence rate of hospital acquired pulmonary infection is apparently increasing. Particularly, pulmonary infection of this kind has characteristics of easily recurrent seizures, long treatment period and high drug-resistance. Hence, it is required for medical personnel to summarize, analyze and study not only general nursing, airway nursing and prevention of pulmonary infection, but also antibiotic selection as well as how and when to use those drugs after the incidence of pulmonary infection for the benefit of patients with indwelling tracheal tube. This article is based on a case collected from Comprehensive Surgery Department of the Third Affiliated Hospital of Inner Mongolia Medical University. The patient's history is as follows: 1. Pulmonary infection; 2. Indwelling tracheal catheter after tracheotomy; 3. Carbon monoxide toxic cerebrosis, mute state. By means of this case analysis, it is expected to make an early detection, and give an early and proper treatment to patients with pulmonary infection caused by indwelling tracheal tubes in clinical practice.
\end{abstract}

Key Words: Indwelling tracheal tube, Nosocomial infection, Pulmonary infection

\section{Medical record}

\subsection{General Information}

The male patient, 50 years old, was admited to hospital in March, 2016. The major causes of admission were "in mute state for 6 years, repeated cough and expectoration for 5 years, recurrence with fever for 2 days". The patient got attacked by acute carbon monoxide toxic cerebrosis due to work 6 years ago, and he was sent and admitted to our hospital to get emergency treatment in MICU. The patient was given bilateral decompressive craniectomy and tracheotomy due to cerebral edema and respiratory failure, and in the end he stayed in mute state after hyperbaric oxygen therapy etc. The intubation was given for a long time because of respira- tory muscle weakness, which caused nasopharyngeal muscles to fail to assist respiration, therefore, the patient needed indwelling tracheal tube for a long term. In the past 5 years, the patient had been suffering from repeated cough and expectoration with an increasing amount of sputa. He was once given treatment in ICU and Comprehensive Surgery Department for multiple hospitalizations. 2 days earlier, the patient began to cough due to cold, with expectoration exacerbated, and the color of sputa was yellow-green and grayish, the body temperature reached up to $38.5^{\circ} \mathrm{C}$ with nocturnal asthma. Amoxicillin, cefaclor and other medicines were given for peroral treatment with poor effects. In the past six years, the patient had been in mute and bedridden state, and was under treatment (passive movement, low-frequency

\footnotetext{
*Correspondence: Shuna Shen; E-mail: shunashen@126.com; Address: Comprehensive Surgery Department, The Third Affiliated Hospital of Inner Mongolia Medical University, China.
} 
electrical stimulation etc.) given by a physical therapist. He was with an indwelling tracheal tube and an indwelling gastric tube. The frequency and the condition of defecation and urination were normal. Past medical history: denial of infectious diseases (hepatitis, tuberculosis etc.); with a history of allergy to penicillins, with a doubtful positive reaction to piperacillin and sulbactam in June, 2012. In 2012, it was effective to present an intravenous fluid therapy of piperacillin-tazobactam and imipenem-cilastatin for the treatment of pulmonary infection, with no allergic reaction. In 2015, intravenous fluid therapy of cefoperazonesulbactam was repeatedly performed to the patient for the treatment of pulmonary infection and upper respiratory tract infection, with no allergic reaction.

\subsection{Physical examination}

$\mathrm{T} 38.5^{\circ} \mathrm{C}, \mathrm{P} 110$ per min, R 22 per min, BP 105/65 mmHg, mute state, and the patient could open his eyes and sense sounds. He was in passive posture and could not cooperate with physical examination. No generalized lymphadenopathy. Old surgical scars in the shape of a question mark were visible on bilateral temples; no edema on eyelids, bilaterial pupils were of equal size and perfect circle with normal reaction to lights; no cyanosis on oral lips, pharynx with mild edema, bilateral tonsils with mild adenoids, no abnormal nasal secretions; soft-neck, trachea was in the midline with indwelling trachea tube, coughing up of yellow sputa. Bilateral lung breath sounds rough, rhonchi and moist crackles on lower double lung, wheezing sounds on the left middle lung occasionally. Heart rate 110 per minute, regular, heart sounds powerful, no murmurs on heart valve auscultation areas; belly flat, no gastrointestinal type and peristaltic wave, abdomen soft with impalpable liver, spleen and subcostal, no abdominal wall resistance, negative shifting dullness, normal peristaltic sounds; no edema on double lower limbs. Neurological examination showed the presence of algesia and topesthesia, 2-grade myodynamia, higher muscular tension, and positive pathological signs on double lower limbs.

\subsection{Auxiliary examination}

Routine blood test: WBC $9.23 \times 10^{9} / \mathrm{L}, \mathrm{N} \% 83.21 \%$, L\% $12.4 \%$, EO $\% 0.1 \%$, RBC $4.18 \times 10^{12} / \mathrm{L}, \mathrm{PLT} 171 \times 10^{9} / \mathrm{L}$, HGB $131 \mathrm{~g} / \mathrm{L}$. Hs-CRP $27 \mathrm{mg} / \mathrm{L}$.

Two sputum culture tests suggested the presence of Pseudomonas aeruginosa.

According to the first antimicrobial susceptibility test, Pseudomonas aeruginosa was susceptible to: cefoperazonesulbactam, levofloxacin, ceftazidime, imipenem, meropenem, piperacillin, piperacillin-tazobactam, cefepime, amikacin, ciprofloxacin; resistant to: tobramycin, gentamicin.

After 3-week anti-infective therapy, according to the second antimicrobial susceptibility test, Pseudomonas aeruginosa was susceptible to: cefoperazone-sulbactam, ceftazidime, imipenem, meropenem, piperacillin, piperacillintazobactam, amikacin; resistant to: levofloxacin, tobramycin, gentamicin, cefepime, ciprofloxacin.

No obviously abnormal signs were in routine urine test, routine stool test and faecal occult blood test. HB5-, HCV-, VDRL-, AIDS-associated antibodies were in the normal range. No abnormal signs were in blood electrolyte analysis, LFT and RFT.

Chest CT Scan: emphysema, bilateral interstitial lung lesions. Bilateral lower lobe pneumonia, right lower lobe long-standing lesion.

\subsection{Preliminary diagnosis}

(1) Pulmonary infection;

(2) With indwelling tracheal tube after tracheotomy;

(3) Carbon monoxide toxic cerebrosis, mute state.

\subsection{Treatment procedures}

The patient had been in bedridden state for a long term with low immunity, recurrent pulmonary infection and indwelling tracheal tube. Because peroral treatment of antibiotics was not effective, on the first day of hospitalization, oxygen inhalation, enhancing back-slap expectoration and empirical use of broad-spectrum antibiotics (cefoperazonesulbactam $3 \mathrm{~g} /$ time, one intravenous fluid therapy every 12 hours) were combined with intravenous fluid therapy of ambroxol and nebulization of budesonide and terbutaline as part of treatment for phlegm-resolving and bronchodilatation. As the symptoms were not relieved after 3-day antiinfective therapy with cefoperazone-sulbactam, the dosage of which was increased (cefoperazone-sulbactam $3 \mathrm{~g} /$ time, one intravenous fluid therapy every 12 hours). However, the patient showed no improvements in symptoms and signs after 5-day anti-infective therapy. According to sputum culture results, etimicin $(100 \mathrm{mg} / \mathrm{time}$, one intravenous fluid therapy every 12 hours) was added and combined with intravenous fluid therapy of aminophylline for bronchodilatation to relieve asthma, occasionally diuretic was used to relieve pulmonary edema. The patient showed no fever and a decrease in the amount of sputa after 2-day anti-infective therapy with cefoperazone-sulbactam and etimicin, and the treatment was effective. After 2-week anti-infective therapy, the patient showed no edema, the amount of sputa either increased or decreased, sometimes with coughing up of yellow, yellow-green and grayish sputa, less rhonchi and moist crackles on bilateral lungs occasionally. Re-examination 
of routine blood test: WBC $6.23 \times 10^{9} / \mathrm{L}, \mathrm{N} \% 73.44 \%$, L\% $17.73 \%$, EO $\% 0.5 \%$, RBC $3.99 \times 10^{12} / \mathrm{L}$, PLT 252 $\times 10^{9} / \mathrm{L}, \mathrm{HGB} 125 \mathrm{~g} / \mathrm{L}$. Hs-CRP $13 \mathrm{mg} / \mathrm{L}$. It was effective to use cefoperazone-sulbactam and etimicin, after 2-week treatment, routine blood test and Hs-CRP indicated the presence of infection. According to sputum culture results, it was changed to intravenous fluid therapy with piperacillintazobactam for anti-infective treatment. It was showed that the amount of sputa was gradually decreased, secretions from indwelling tracheal tube reverted to white, cough was relieved, no dyspnea and asthma. After 1-week intravenous fluid therapy of piperacillin-tazobactam, patient showed no cough, expectoration, asthma, sweat and face-flushing etc. In the re-examination of routine blood test, WBC, NEUT and LYM etc. were in the normal range, Hs-CRP $5.8 \mathrm{mg} / \mathrm{L}$. The patient's condition had been apparently improved, and general nursing of indwelling tracheal tube and generalized physical therapy were required and continued. After discontinuation of anti-infective drugs for one month, the patient still kept in stable condition.

\section{Discussion}

\subsection{Dr. Jin Guo}

Dr. Jin Guo, Primary-Care Doctor, Attending Doctor, Comprehensive Surgery Department of The Third Affiliated Hospital of Inner Mongolia Medical University.

After admission, according to patient's medical history, vital signs and auxiliary examinations, it was definitely diagnosed as pulmonary infection, combined with indwelling tracheal tube and carbon monoxide toxic cerebrosis etc., hence, the patient was susceptible to recurrent pulmonary infection; as the patient had been in hospitalized and bedridden state for a long term, the pulmonary infection of this time was considered as hospital acquired infection. Sputum culture results indicated the presence of Pseudomonas aeruginosa, which is one of the common nosocomial infectious bacteria and multidrug-resistant bacteria. ${ }^{[1]}$ It was required to perform combination therapy and intravenous fluid therapy for anti-infective treatment. ${ }^{[2,3]}$

According to diagnostic criteria presented in "Diagnosis and Treatment Guidelines of Hospital-Acquired Pneumonia" formulated by Chinese Thoracic Society, the bacteriological diagnostic criteria of nosocomial pulmonary infection with Pseudomonas aeruginosa are as follows: (1) Predominant Pseudomonas aeruginosa is isolated from more than 2 continuous sputum cultures. (2) Blood culture results are identical to that of sputum culture. (3) Pseudomonas aeruginosa can be isolated from the culture of pleural effusion. (4) When sputum culture shows negative results or above examinations are difficult to identify whether it is bacterial infection or bacterial colonization, fibrobrochoscope examination should be performed to collect airway secre- tions or tissue specimen for baterial culture.

These 2 sputum culture tests for this patient were exercised by collecting sputa from trachea through the indwelling tracheal tube, and the results showed the presence of Pseudomonas aeruginosa. Routine blood test suggested the incidence of severe bacterial infection, chest CT scan suggested bilateral lower lobe pneumonia (conforming to imaging characteristics of hypostatic pneumonia caused by longterm bedridden condition), definitely diagnosed.

Antibiotics should be applied according to sputum culture results, it was ineffective to use single antibiotic of cefoperazone-sulbactam in the treatment of nosocomial pulmonary infection, and combined use of antibiotics was required.

\subsection{Dr. Cuiping Yan}

Dr. Cuiping Yan, Former Nurse-In-Charge of General Surgery Department, Head Nurse, Comprehensive Surgery Department, The Third Affiliated Hospital of Inner Mongolia Medical University. Research interest: Observation of effects of high-quality nursing on controlling nosocomial infections in postoperative patients.

Nosocomial infection is a way for pathogens to invading into human bodies. Under the condition of immune defense mechanism and immune function weakened, infectious pathogens grow and multiply in human bodies, and eventually produce metabolites, which are harmful to host cells and functions of human tissues. ${ }^{[4]}$ Pathogenic factors inducing nosocomial infections mainly include poor understanding of nosocomial infection and its hazards, failure in strictly enforcing aseptic operation and disinfection $\&$ isolation regulations, deficiency in effect monitoring of disinfection and sterilization and other objective factors, along with subjective factors such as invasive operations, excessive use of hormones or immunosuppressants and excessive use or improper use of antibiotics. ${ }^{[5]}$ High-quality nursing service refers to a modernized nursing mode, which is patient-oriented and guided by modern nursing service concept, to promote nursing service by strengthening basic nursing, fully implementing nursing responsibility system and deepening nursing exploration. High-quality nursing service includes: (1) Ward environmental management: wards are required to be ventilated everyday to guarantee fresh air and soft lights, and keep wards quiet, tidy and comfortable; wards must be disinfected periodically every month. (2) Infection monitoring: Strictly carry out disinfection and segregation regulations, medical personnel must strictly observe aseptic operation; bacteriological detection must be conducted for medical instruments and disinfectants before use. (3) Enhancing basic nursing: Intensive monitoring of special patients should be strengthened. It is necessary to guarantee basic nursing, such as regular turn- 
over, massages, hand washing, bedclothes replacement and hygiene inspections. (4) Tube care: It is necessary for patients with indwelling catheters to keep tubes unobstructed and avoid the incidence of tube wrinkling and tube obstruction caused by sputa; It is required to strictly disinfect indwelling tracheal tube 2 times/day. (5) Usage management of antibiotics: It is required to strictly follow the results of antimicrobial susceptibility test and physicians' instructions to give drugs to patients who need to use antibiotics, it is prudent to use broad-spectrum antibiotics to avoid the generation of multidrug-resistant bacteria. (6) As for patients with pulmonary infections, it is required to guarantee nursing treatments, such as airway patency, low flow oxygen inhalation, thorough nebulization for phlegm-resolving, regular turn-over and back-slap for expectoration and timely sputum suction with tube. Be aware of oral care, decrease and avoid secretion inhalation.

\subsection{Dr. Shuna Shen}

Dr. Shuna Shen, Master Supervisor, Chief Physician, Comprehensive Surgery Department, The Third Affiliated Hospital of Inner Mongolia Medical University. Research interest: How to prevent postoperative infections effectively.

Pathogens causing hospital infections in internal medical departments are mainly gram-negative bacteria, ${ }^{[6]}$ followed by fungi and gram-positive bacteria. Gram-negative bacteria, which are opportunistic pathogens and widely spread in various hospital environments and medical instruments, can cause hospital infections by medical personnel with contaminated hands and invasive operations. Hence, it is imperative that medical personnel not only comply with hand hygiene, but attach importance to cleaning and disinfection management of environments and medical materials. Pathogens causing hospital infections, with top-ranking detection rates in hospitals, are as follows: Klebsiella pneumoniae, Pseudomonas aeruginosa, Candida albicans and Staphylococcus aureus. Meanwhile, the distribution and drug resistance of pathogens vary with regions and hospitals. Taking the practical situation of hospital infections into account, medical personnel in internal medical departments are required to arise awareness of proper and rational use of antibiotics.

Nosocomial infections are common in patients with chronic cardiopulmonary disease, long-term use of drugs (antibiotics, hormones and anti-cancer drugs) and low immunity. Critically ill patients show apparent toxic symptoms such as shivering, hyperpyrexia etc., and part of patients show symptoms of relative bradycardia, cough and expectoration of yellow (even yellowish green for typical sufferers) phlegm. Other symptoms include dyspnea, progressive cyanosis, even respiratory failure and peripheral circulatory failure for seriously ill patients. Patients with nosocomial infections also show atypical symptoms.
In the past 3 years, the patient mentioned above had been using antibiotics repeatedly, and one of the most frequently used antibiotics was cefoperazone-sulbactam. The sputum culture results showed the presence of Pseudomonas aeruginosa. Although it is susceptible to cefoperazone-sulbactam, the susceptibility is not high due to repeated use.

\subsection{Dr. Chunyan Qu}

Dr. Chunyan Qu, Chief Physician, Comprehensive Surgery Department, The Third Affiliated Hospital of Inner Mongolia Medical University. Research interest: Lemology and rational use of antibiotics.

Pseudomonas aeruginosa pneumonia can be subdivided clinically into two types: bacteraemic pneumonia derived from bloodborne pathogens or mononuclear phagocytes; and non-bacteraemic pneumonia caused by inhalation of upper respiratory tract secretions. Pneumonia caused by Pseudomonas aeruginosa usually happens to patients with low immunity or underlying diseases. Risk factors inducing infections with Pseudomonas aeruginosa are as follows: recent hospitalization; frequent use of antibiotics (4 times in a year) or antibiotic use in recent 3 months; severe COPD; Pseudomonas aeruginosa isolated in the past acute exacerbation or presented in the stable phase. Pseudomonas aeruginosa pneumonia, one of the most common hospital-acquired pneumonia, difficult to be cured with high case fatality rate, has been a chronic and stubborn disease among clinical pulmonary infections. A large amount of clinical controlled trials and retrospective studies ${ }^{[7]}$ show Pseudomonas aeruginosa are susceptible to following antibiotics: piperacillin-tazobactam, etimicin and imipenem; and drug susceptibilities are all above $80 \%$. The antibiotic among third-generation cephalosporins which affect Pseudomonas aeruginosa most is Fortum (ceftazidime), but the drug susceptibility is only $60 \%$. The susceptibility of Pseudomonas aeruginosa to ceftizoxime is 55\%. Pseudomonas aeruginosa is almost resistant to cefoxitin and ampicillin because of their low susceptibilities, therefore, they cannot be used in the treatment of Pseudomonas aeruginosa pneumonia. The susceptibility rates of Pseudomonas aeruginosa to amikacin and levofloxacin are also in a high level. It is recommended to choose antibiotics which have effects on Pseudomonas aeruginosa to provide an active treatment for patients with Pseudomonas aeruginosa pneumonia. When selecting antibiotics, piperacillin-tazobactam and etimicin are preferentially considered due to their high susceptibilities, and can make a combined use with levofloxacin.

During the early stage of infection, empirical use of cefoperazone-sulbactam was made to the patient mentioned in this case with the aim of giving an anti-infective therapy. Although sputum culture results indicated it was susceptible to cefoperazone-sulbactam, the patient responded poorly to the treatment with minor improvements in clinical symp- 
toms and vital signs. Piperacillin-tazobactam and etimicin (with high susceptibility) were applied to the later treatment, which proved to take good effects as expected. As the patient was hospitalized for a long term, in view of medical history and sputum culture, the patient had a nosocomial pulmonary infection. Hence, a thorough anti-infective therapy was recommended. After 3-week anti-infective therapy, the patient's condition had been improved obviously. After discontinuation of anti-infective drugs for one month, the patient still kept in stable condition.

On all accounts, patients with indwelling tracheal tube are susceptible to nosocomial infections, which bring great hazards to lungs and other organs and severely affect the curative ratio in patients with infections. As an enterprise-owned

\section{References}

[1] Wu AH, Wen XM, Li CH, et al. China National Point Prevalence Survey on Healthcare-associated Infection and Antimicrobial Use in 2012. Chinese Journal of Infection Control. 2014; 13(1): 8-15.

[2] Liu JM, Ren H. Investigation and Analysis of Nosocomial Infections in Hospitalized Patients of Internal Medicine Department. Chinese Journal of Nosocomiology. 2013; 23(16): 3848-3849.

[3] Ministry of Health, PRC. Regulations on Hospital Infection Administration. Ministry of Health, PRC. 2000; 15-20.

[4] Ministry of Health, PRC. Standards for Diagnosis of Nosocomial Infections (Trial) - Excerpt (1). Journal of New Medicine. 2005; 36(8): hospital, our hospital has admitted a large amount of industrial injured patients of this kind and patients with tracheal intubation, which need a long-term hospitalization. In order to provide proper prevention and treatment methods, it is imperative for medical personnel and nursing staff to make a further prevention and treatment education with the aiming of taking appropriate prevention measures, timely discovering nosocomial infections, developing reasonable and individualized treatment plans, early intervening, preventing complications, increasing curative ratio and shortening hospitalization periods.

\section{Conflicts of Interest Disclosure}

The authors have no conflicts of interest related to this article.

495 .

[5] Xu XY, Zhang Y, Shi YP. Analysis of Nosocomial Bacterial Infection and the Risk Factors among Patients in Respiration Internal Medicine. Modern Preventive Medicine. 2014; 41(4): 763-764.

[6] Dereli N, Ozayar E, Degerli S, et al. Three-year Evaluation of Nosocomial Infection Rates of the ICU. Revista Brasileira de Anestesiologia. 2013; 63(1): 79-84.

[7] Pan J, Li R, Zhou YS, et al. Analysis in Curative Effect of Piperacillin-tazobactam Combined with Amikacin on Pandrugresistant Pseudomonas Aeruginosa Pneumonia. Chinese Journal of Traditional Medical Science and Technology. 2014(s2): 161-161. 